\title{
Guía Práctica para Desarrollar un Programa Académico: el Caso de la UNED de Costa Rica
}

\author{
(Practical Guide to Develop an Academic Program: Costa Rican UNED’s Experience)
}

\author{
Alejandra Cruz \\ GUISELLE HIDALgo \\ AnABelle Castillo \\ Universidad Estatal a Distancia (UNED) \\ (Costa Rica)
}

RESUMEN: La Universidad de la sociedad de fines del siglo XX, está presionada a adecuarse a los cambios que la sociedad posmoderna le imponen. Por otra parte, aun cuando las instituciones de educación superior no se hayan preparado para enfrentar estos retos y no tengan desarrollada una cultura evaluativa, están en la obligación de ir habilitando estas transformaciones oportuna y efectivamente. Considerando estos parámetros, el presente artículo ofrece un modelo que permite implementarse en un plazo relativamente reducido y acorde con las exigencias indicadas. Asimismo, se presentan los resultados de su aplicación en una universidad estatal a distancia, cuyo objetivo es mejorar un programa académico. Se trabaja con los programas académicos debido a que estos son la forma concreta para que la universidad se adapte a los cambios del mundo y se realiza bajo la perspectiva de aspectos de calidad que los mismos siempre deben tener.

Universidad a Distancia - Habilidades Estudiantiles en un Programa de Negocios - Modelo de Evaluación de un Programa Académico - Nuevas

Formas de Educación en la Sociedad

ABSTRACT: This is a moment for big changes. The end of XX Century society is under extreme pressures to adapt itself to the new rules of Postmodernist Era. In the middle of this storm, we find universities trying to understand, analyze and take new ways in this new society. Although, superior education institutions haven't been prepared for facing those challenges, they should be in way to them by evaluating their system and adapting to the new circumstances. The present article offers a model that permit, in a short period of time, to execute changes in an academic program. Specifically, it is the result of a research being carried out for a business program of a distance education university. It focus at the importance of evaluating academic programs because those represent the final result of a university effort, they are the specific instruments universities have to meet their changes, to meet their quality standards and to offer students all the best that have been planed.

Distance Education University - Student's Skills in a Business Program - Evaluation Model of Academic Program - New Education's Ways in the Society 


\section{INTRODUCCIÓN}

La sociedad de fines del siglo XX es una sociedad en constante cambio que ejerce gran presión sobre sus instituciones para adaptarse a sus exigencias. Una de las más afectadas por estas nuevas demandas son las instituciones de educación superior, de las cuales se requiere tanto una adecuación de sus contenidos acorde con las nuevas necesidades como mayores exigencias en cuanto a calidad.

Escotet resume en la siguiente afirmación la responsabilidad de la Universidad: "el eje de acción universitaria y en general de la educación: formar al hombre para el cambio permanente y aún para la eventual crisis producto de la transición" y, continúa diciendo: "Es decir, que la mejor ley sobre la Universidad es aquella cuyo único articulado la obliga a cambiar sin pausa y no a ir por detrás de los cambios". (Escotet, 1992:51)

Para la evaluación del quehacer universitario se tiene una gran diversidad de enfoques que difieren en dimensión, profundidad, cobertura e incluso en la misma metodología empleada. La dimensión mayor cubriría en su totalidad las actividades institucionales, y se refiere a la evaluación institucional (Espinoza y otros, 1994). En el caso particular del estudio que aquí se describe, se circunscribe a probar un modelo teórico de evaluación con un programa académico específico impartido a distancia y el objetivo principal es presentar algunas contribuciones prácticas que se traduzcan en el mejoramiento de dicho programa con sus diferentes énfasis.

Por otro lado, para que las recomendaciones de un estudio evaluativo produzcan resultados efectivos, dependerá en gran medida de si se tiene o no una cultura evaluativa, pues de otra forma, "la resistencia a la evaluación genera conductas evasivas de parte de todos los actores del proceso docente, que se traducen en manifestaciones de escepticismo frente a la intención de mejoramiento que suelen sostener un número reducido de académicos." (Ahumada, s.f.).

Jacques L'Ecuyer (L'Ecuyer, 1997) tipifica algunos de los argumentos utilizados por estas organizaciones, resistentes a la evaluación, de la siguiente manera:

u La evaluación es una infracción a su autonomía

Es una manera de imponer nuevos modelos

Esconden sus problemas y se muestran ellos mismos como los mejores

Sin embargo, a pesar de la resistencia, se debe tener presente que la tendencia mundial actual hacia la globalización de las economías, compromete a las universidades a producir profesionales competentes que respondan a esas exigencias y den ventaja competitiva a sus respectivos países. La certificación pública de su calidad se viene logrando por la vía de la acreditación de sus programas, ya sea con base en una autoevaluación supervisada externamente con pares externos (académicos de amplia experiencia en el campo disciplinario respectivo, instituciones nacionales o del exterior, o profesionales experimentados y reconocidos dentro de la Educación Superior) (CONARE-OPES, 1996 ${ }^{\mathrm{a}}$ o por medio de una evaluación externa. A la par de esto, surgen las presiones sociales que obligan a las universidades a dar cuenta de su efectividad, especialmente sobre aquellas que emplean fondos públicos. También se reafirma el punto de que esta obligación es independiente a su autonomía, y que ésta no la libera de la responsabilidad de dar cuentas a la sociedad.

Ahora bien, la forma concreta como las Universidades se adaptan a las demandas del cambiante mundo, es por medio de la modificación de sus programas. Como bien lo resume L'Ecuyer, "Los programas de estudio constituyen realmente el corazón de la misión de las universidades. Por sus 
programas, las instituciones transmiten a sus estudiantes los conocimientos que necesitan, desarrollan sus capacidades y les inculcan principios y actitudes... Es decir, que el programa es realmente el proceso central de la universidad” (L’Ecuyer, 1997:1)

Lo importante entonces es buscar métodos efectivos que permitan adaptar los programas a las nuevas demandas de la sociedad. Es necesario dar respuestas rápidas a los cambios, sin perder de vista que los programas deben responder en todo momento a las exigencias de calidad.

El primer paso en el proceso de adecuación de estos programas es la evaluación de los mismos. El tema de la evaluación cobra así trascendental importancia, puesto que no todos los modelos pueden adaptarse a las necesidades de la sociedad moderna, por el contrario, una evaluación lenta puede conducir a obtener resultados poco actualizados, e incluso puede estar relacionada con situaciones inexistentes, puesto que las necesidades que el cambio son tan evidentes que se dan aun sin esperar los resultados de la evaluación.

Por tal razón, el presente trabajo ha tratado de encontrar un método de evaluación que se plantee todas estas necesidades, que ofrezca soluciones a una sociedad cambiante, pero que no deje de lado los aspectos medulares de una verdadera evaluación.

Como este trabajo es el producto de una investigación realizada en la Escuela de Administración de Empresas de una universidad a distancia: la UNED de Costa Rica.. Esto impone ciertas particularidades al caso que se presenta, por lo que algunas recomendaciones son propias de la educación a distancia. Sin embargo, el producto de este estudio es una guía para desarrollar un programa que puede ser aplicable a cualquier institución de educación, sea esta a distancia o no.

\subsection{LA UNIVERSIDAD ESTATAL A DISTANCIA (UNED) DE COSTA RICA}

La UNED cumplió en 1997 veintidós años de su creación ${ }^{6}$. La forma como dio sus primeros pasos y su posterior desarrollo, ha generado en la práctica un gran cúmulo de experiencias sobre educación a distancia entre sus funcionarios.

Sin embargo, cabe destacar dentro de esa experiencia acumulada, ciertas particularidades con respecto a la forma en que se ha venido desarrollando el modelo de educación a distancia. En primer lugar, hay que señalar que la manera como se concibió la educación a distancia y la forma como se organizó la universidad desde sus inicios, separó la acción académica-docente de la académica-investigativa. Se crearon dependencias separadas para funciones académicas concebidas como separadas. Lo académico se organizó en procesos, dentro de un sistema que responsabilizaba en diferentes instancias u oficinas, las actividades interrelacionadas e independientes del proceso instruccional (la concepción, el diseño, la producción y la conducción de los cursos). Con el tiempo se han realizado esfuerzos para solventar esta disfunción, pero las raíces conceptuales han sido más fuertes que los cambios organizacionales que se han ejecutado.

Por otra parte, si analizamos la situación actual en la UNED, se puede observar por medio de un estudio reciente sobre los medios disponibles y las formas de evaluación del rendimiento del estudiante en nuestro sistema educativo (Ramírez H, 1997) que, únicamente el 8\% de los cursos analizados

\footnotetext{
${ }^{5}$. La investigación se titula: Cruz, A.., Hidalgo, G. y Castillo, A. "Propuesta para mejorar el programa de administración de empresas”. UNED, 1997.

${ }^{6}$ La UNED fue creada por ley \# 6044 en 1977.
} 
utilizan como medio de apoyo el material audiovisual, un 40\% utilizan tan solo libro. Además, en la escuela de Administración, un 70\% de los cursos optaron por seis tutorías presenciales no obligatorias, y la tutoría telefónica tiene un grado de baja importancia en el 77\% de los cursos.

Además, en este mismo estudio se determina que la evaluación se basa en un $80 \%$ y más en exámenes y quices para el $66 \%$ de los cursos ofrecidos de esta escuela. Si a esto se agrega que la UNED no ha incorporado el medio de apoyo telemático a su paquete instruccional, cabe preguntarse, si con estas condiciones, se está cumpliendo con las necesidades de formación integral, vivencial, de conocimiento interno de la empresa que requieren los profesionales.

\subsection{LA ESCUELA DE CIENCIAS DE LA ADMINISTRACIÓN}

La Escuela de Ciencias de la Administración de la UNED cuenta para la oferta académica de 1997 con los siguientes énfasis:

1) Administración de Empresas (con nivel de diplomado).

2) Administración de Empresas con concentración en Gestión Organizacional.

3) Administración de Empresas con concentración en Empresas Cooperativas y Asociativas.

4) Administración de Empresas con concentración en Contaduría.

5) Administración de Empresas con concentración en Banca y Finanzas.

El diplomado de Administración es común para todas las concentraciones o énfasis que se ofrecen en los programas de las carreras, a saber de los énfasis 2) al 5) antes señalados.

A partir de 1990 la escuela vive una apertura, otorgando una variedad de grados académicos, así como un reacomodo de sus énfasis. A partir de ese año, debido a cambios internos en la UNED, se observa una flexibilización en el cierre y apertura de programas.

Con respecto a la población estudiantil es importante destacar que la matrícula de esta escuela tuvo un aumento a partir de 1992 y esta se ha mantenido hasta 1998. Por otra parte, con respecto a los graduados se puede indicar que para junio de 1997 existe un total de 1716 graduados $^{7}$, abarcando desde los pregrados académicos de técnico y diplomado, hasta los grados académicos de bachiller y licenciatura.

\section{HACIA LA SELECCIÓN DEL MODELO DE EVALUACIÓN}

Como se ha dicho anteriormente, el primer paso para adaptar un programa a las necesidades cambiantes de la sociedad moderna es evaluarlo, por lo que surge la pregunta de cuál debe ser el modelo de evaluación apropiado.

Las primeras reflexiones nos llevan a afirmar que se hace necesario realizar una evaluación integral. Sin embargo, un análisis más profundo nos lleva a la conclusión de que esto no siempre en posible.

Por lo anterior, el pensamiento que guió la investigación fue buscar el fortalecimiento de lo sustancial debido a la imposibilidad de hacer un cambio integral, y por ello se partió de los siguientes lineamientos y así poder orientar este trabajo investigativo:

7. Fuente: Oficina de Registro (cuadro: Total de alumnos graduados 97-I por título y carrera al 6 de junio de 1996) y el "Informe de labores del rector 1996", p.100. (UNED, 1996c). 
No se puede cambiar todo.

u Se debe empezar por lo sustancial.

u Determinar lo "clave" dentro del programa para fortalecerlo.

\subsection{CONSIDERACIONES ACERCA DE LA INTEGRALIDAD DEL ENFOQUE}

La evaluación integral de un programa académico, conduce a determinar tanto la efectividad de los métodos pedagógicos para lograr los objetivos de aprendizaje en los estudiantes como la pertinencia del mismo para la sociedad. Ambos aspectos son igualmente importantes.

Definida de esta manera, surge la necesidad de evaluar tanto los contenidos, como el conjunto de medios didácticos para lograrlos. La evaluación debería determinar entonces qué tan aptos son esos medios didácticos para alcanzar los objetivos propuestos en el programa, en qué medida toman en cuenta las características de los estudiantes y si esos objetivos cumplen con las expectativas planteadas por la sociedad.

Sin embargo, surge un problema práctico, si la evaluación determina que los objetivos y el contenido de los programas no cumplen con las expectativas planteadas por la sociedad o la institución que los fijó, sería de poca utilidad investigar la efectividad de los medios didácticos que se están dando para favorecer esos objetivos específicos. La conclusión inmediata, es entonces, evaluar en primera instancia los objetivos y contenidos antes que los medios.

\subsection{CRITERIOS ESPECÍFICOS DEL MODELO DE EVALUACIÓN}

Para reforzar el argumento planteado en el punto anterior, podemos ver cómo, a través del tiempo, la calidad de un proceso evaluativo ha tenido diferentes énfasis. Según Ahumada, P. (1996), inicialmente interesaba centrar el proceso en QUÉ ENSEÑAR, dando importancia en los fines y los medios. Posteriormente, interesó el cómo ENSEÑAR, es decir, con qué recursos tecnológicos se cuenta para el apoyo docente. Hasta hace poco tiempo, interesó CUANTO APRENDIÓ, es decir, ligado a los resultados del proceso, y con ello los criterios y procedimientos de evaluación son los que demanda mayor atención. Actualmente, interesa averiguar POR QUÉ Y PARA QUÉ SE ENSEÑA TAL O CUAL CONTENIDO, es decir, analizar los objetivos y contenidos de los programas frente a las necesidades que la sociedad requiere.

Podemos resumir hasta aquí el modelo que se ha venido construyendo, como aquel que toma en cuenta los objetivos y contenidos y que a la vez evalúa esos contenidos a la luz de las necesidades de la sociedad. Por lo tanto, el paso a seguir sería establecer los criterios específicos que nos conduciría a tal evaluación. Para tal efecto se consideró que los criterios de calidad planteados por Jacques L'Ecuyer vendrían a completar este modelo. L'Ecuyer centra su atención en la eficacia del programa y presenta un desglose de aspectos a considerar que ofrecen una visión de todo cuanto debe evaluarse alrededor de los criterios aquí establecidos, a saber: por qué y para qué enseñar. A continuación se describen:

u Pertinencia del programa: Se analiza que los objetivos, normas y el contenido del programa de estudios sean acordes con las expectativas y las necesidades del mercado de trabajo, la sociedad, los estudiantes o de las universidades.

u Coherencia del programa: Se observa que los objetivos del programa describan claramente las competencias por desarrollar, las normas establecerán los niveles o grados a las que dichas competencias deben ser dominadas en la educación. Además, las actividades de aprendizaje deben ser ordenadas lógicamente y sus exigencias deben ser establecidas en forma clara y realista. 
u Valor de los métodos pedagógicos y del apoyo a los estudiantes: Se analiza que los métodos pedagógicos se adapten a los objetivos del programa y a cada una de las actividades de aprendizaje.

u Adecuación de los recursos humanos, materiales y financieros: El personal profesional y de apoyo es suficiente y posee las calificaciones necesarias para responder a las necesidades del programa.

Eficacia del programa: Los modos y los instrumentos de evaluación de los aprendizajes aplicados en el programa permiten evaluar la realización de los objetivos asignados a las actividades de aprendizaje y al programa.

u Calidad de la gestión del programa: Se considera que los procedimientos sean claros y ayudan a evaluar regularmente, mediante datos cuantitativos y cualitativos confiables, las fortalezas y debilidades del programa y cada una de las actividades de aprendizaje. La descripción del programa de estudios se encuentra debidamente distribuida y explicada a los estudiantes así como a los profesores concernidos.

Una vez definidos los criterios de evaluación, el paso siguiente fue determinar cuáles son esas demandas que la sociedad plantea para que el programa en estudio cuente con los criterios de calidad ya expuestos, en el caso que nos ocupa, un programa de administración de empresas.

\section{3 . MODELO DE ANÁLISIS}

En el esquema siguiente se resume el modelo de análisis que se utilizó en la investigación. En éste se observa cómo las destrezas son el punto de integración entre las columnas de la SOCIEDAD y la UNIVERSIDAD. La importancia que esas destrezas tienen para que las empresas cuenten con el perfil de gerente moderno idóneo, son las mismas que la universidad debe tener en los contenidos de sus programas para poder ofrecer un perfil de graduado, consecuente con las necesidades que requieren las empresas modernas dentro de las condiciones de la sociedad actual.

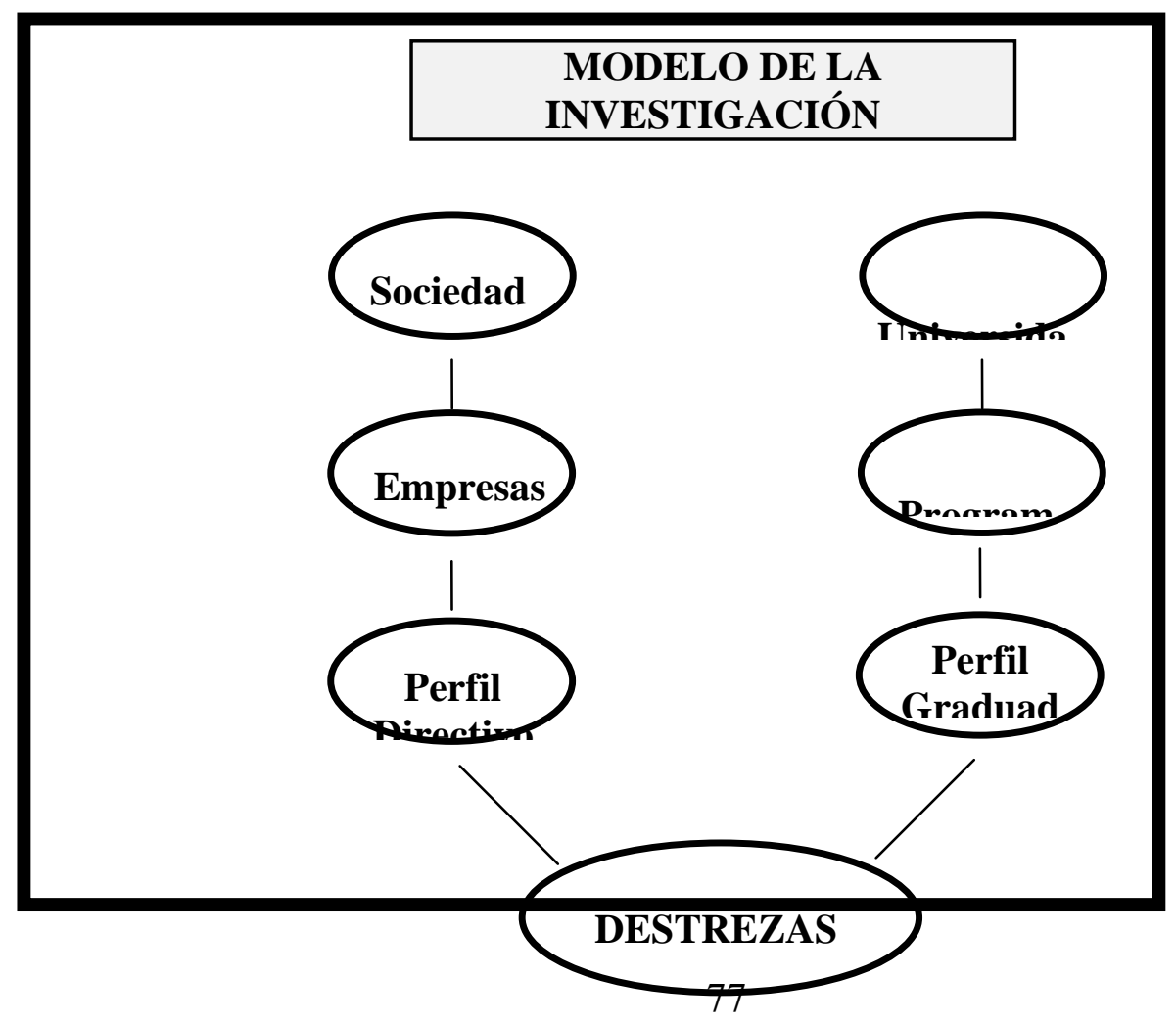




\section{Diagrama 1}

El modelo teórico de investigación aquí planteado es bastante simple, ofrece puntos medulares que pueden ser aplicados en diferentes medios y a carreras diversas. Lógicamente, cada aplicación puede requerir caminos metodológicos un tanto diferentes.

A continuación se ofrece como ejemplo la aplicación del mismo a la carrera de Administración de Empresas de la Universidad Estatal a Distancia en Costa Rica.

\section{APLICACIÓN DEL MODELO EN EL CASO DE LA ESCUELA DE CIENCIAS DE LA ADMINISTRACIÓN DE LA UNED}

Para llevar a la práctica este modelo, se procedió a definir los hechos más relevantes que caracterizan a la sociedad actual y que deben ser tomados en cuenta en la definición de los contenidos de un programa académico de administración de empresas.

\subsection{LOS NUEVOS REQUERIMIENTOS DE LA SOCIEDAD}

En esta "Tercera Ola"(Toffler, 1994) que estamos viviendo, la producción es desmasificada, es decir se produce en cantidades pequeñas una gran variedad de productos para satisfacer necesidades específicas de los individuos.

La desmasificación constituye la clave manufacturera de un nuevo sistema productivo que ha venido a revolucionar todos los principios de la administración en nuestros días. Para diversificar la producción, se hizo necesario rediseñar la planta, pasándose del viejo sistema altamente estandarizado y con gran cantidad de trabajo manual, a un sistema altamente automatizado, organizado en talleres, que permitan el ensamblaje de diversos componentes, los cuales resultan en una mezcla de estilos, colores y funciones tal, que produzca la variedad requerida con calidad y bajo costo ( Drucker, 1988).

Pero aun más, para lograr los objetivos propuestos, se requiere apoyar el nuevo sistema productivo con una organización administrativa y de apoyo totalmente diferente a la que se ha venido utilizando hasta el momento. En primer lugar, el empleado de la planta debe estar preparado, no para realizar trabajo manual, sino para trabajar con máquinas altamente sofisticadas que le exigen conocimientos muy especializados. Se requiere entonces, de un funcionario capaz de manejar sistemas de información con alto grado de complejidad, de lograr una autosuficiencia tal que pueda enfrentar y solucionar los problemas que se presenten y por lo tanto creativo, dinámico para responder a los problemas con rapidez y capaz de tomar decisiones cuando las circunstancias lo requieran

La nueva tecnología requiere que individuos bien preparados puedan lograr esta autosuficiencia que redunda en menores costos al reducir el personal de apoyo que se ha venido necesitando alrededor de las funciones de producción, al disminuir los tiempos de producción y finalmente al lograr una menor cantidad de defectos gracias al uso de esa tecnología.(Chase, 1989). 
Para que estos nuevos sistemas puedan funcionar eficientemente, se hace necesario realizar una serie de replanteamientos, tanto en la forma en que se organiza la planta, se distribuyen las nuevas máquinas, como en la implantación de una serie de principios de producción como los de "Justo a Tiempo"(Chase, 1989) que requiere que recursos humanos, equipo y materiales se encuentren en el momento y en el lugar oportuno para evitar las demoras.

Integrado a este sistema de producción se encuentra un nuevo sistema contable que mide costos y explica resultados a partir de conceptos diferentes a los que se han venido manejando. El sistema de costos basado en las mediciones de la mano de obra directa, mano de obra indirecta y materiales a cedido el lugar a un sistema que mide el costo y resultados de las innovaciones, la velocidad con que se produce en el tiempo, la calidad o la ausencia de defectos y el costo de la tecnología (Stalk, 1988 ).Es decir, es un nuevo sistema que mide más que el costo de mano de obra, la inversión en conocimiento hecha en el recurso humano.

El mercadeo es considerado más bien una función integrada al sistema de producción. El mercadólogoingeniero de producción es el que valora las necesidades, gustos y preferencias de los clientes para desarrollar los productos que se ajusten a esas necesidades, pero que a la vez sea un diseño que el sistema de producción pueda trabajar (McKenna, 1991).

El sistema de servicios administrativos debe estar al servicio de la producción, por lo que se requiere de estructuras simples, desburocratizadas, lo cual se puede lograr con la implementación de la tecnología adecuada.

Es decir, si bien antes se necesitaba de una serie de niveles jerárquicos para transmitir y resumir información de la base a la cúspide, hoy la síntesis de ella que se realiza por medio de la computadora, permite reducir drásticamente el número de esos niveles.

Como condición a esta nueva forma de organización administrativa se necesita una alta capacitación del individuo que le permita desarrollar, dominar los sistemas de información y obtener el mayor provecho de ellos para la toma de decisiones oportunas.

\subsection{APLICACIÓN DEL MODELO DE INVESTIGACIÓN}

A partir de la caracterización que se hizo de la sociedad, se procedió a seleccionar las destrezas que requiere el graduado en administración de empresas para enfrentarse a los retos de esta sociedad. Una vez definidas estas destrezas, se seleccionaron los cursos del programa que aportan conocimientos que permiten desarrollar destrezas indispensables para el buen funcionamiento de las mismas dentro de esta sociedad moderna.

Posteriormente a esta definición de cursos claves, se establecieron las estrategias pedagógicas que deberían tener, con el fin de ir definiendo el modelo pedagógico seguir para a su vez lograr las destrezas y conocimientos necesarios.

Es conveniente aclarar que las destrezas han sido seleccionadas a partir de los retos que los cambios tecnológicos y a las transformaciones socioeconómicas imponen y que obligan a las empresas a organizarse de cierta manera para poder sobrevivir ante la competencia. No se trata de establecer un listado exhaustivo de destrezas, sino aquellas indispensables para hacer frente a los nuevos retos de la sociedad. Con ello se pretende lograr una primera aproximación teórica a lo que debe ser un programa académico ajustado a los nuevos retos de la sociedad. 
En el diagrama que se presenta a continuación se sintetiza el resultado de este modelo teórico laboral.

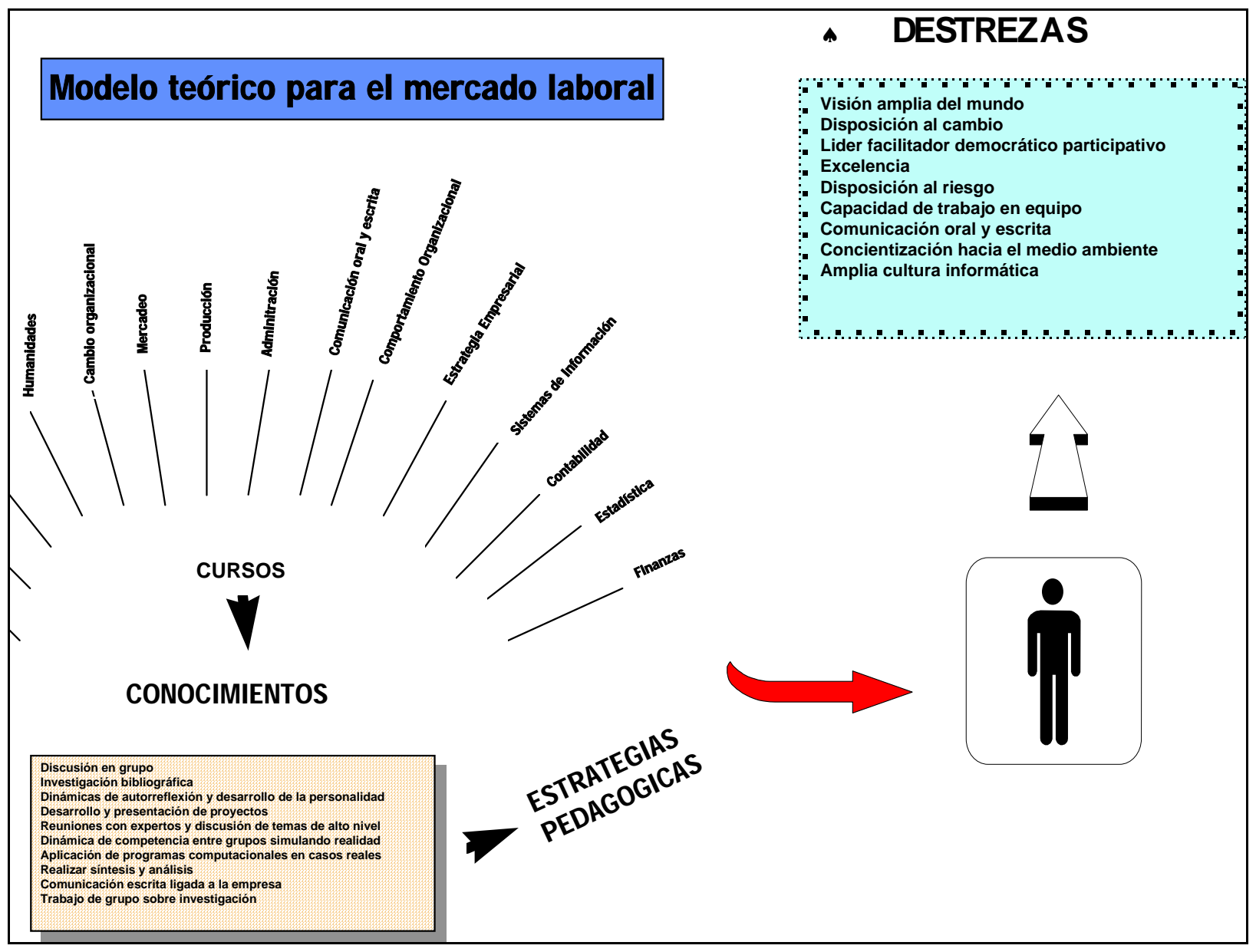

Diagrama 2: Modelo teórico del mercado laboral

\subsection{METODOLOGÍA}

La experiencia previa del equipo de investigación en la aplicación de técnicas de investigación cuantitativa como es el cuestionario, unido a las dificultades propias de la administración de la universidad para realizar una aplicación rápida y eficiente de este tipo de instrumento, influyó en forma determinante en la necesidad de aplicar una técnica que permitiera visualizar y aproximarse a situaciones más reales (Ruiz, 1989: 18) pero que además fuera eficiente y de mayor rapidez para obtener resultados. Por este motivo se acudió a la entrevista en grupo, así como la entrevista individual.

Se entrevistó en forma grupal a empresarios, estudiantes y a dos grupos de funcionarios académicos. La entrevista individual se utilizó en dos ocasiones especiales debido a la relación jerárquica que esas personas mantenían con el grupo de funcionarios académicos, la justificación metodológica se presenta más adelante. 
La entrevista en grupo es una forma de hacer una entrevista cualitativa a profundidad. Para el caso que nos ocupa, se ha escogido lo que Ruiz e Ispizua denominan como tipo \#3 en donde "... se entrevista a un solo grupo, una sola vez, en una sesión más o menos extensa de tiempo" (1989: 160).

Los motivos para seleccionar este tipo de entrevista se resumen con palabras de los autores citados:

"La entrevista en grupo ofrece unas oportunidades de conocimiento y de análisis que la entrevista individual no ofrece o las ofrece en condiciones menos ventajosas.

En primer lugar, el grupo es un gran provocador. La inmensa mayoría de los individuos cuentan con sólo una capacidad muy escasa de autobservación, que se reduce más a la hora de realizar un autoanálisis, y que se ve aún más limitada para articular con claridad y precisión el resultado de sus propias observaciones y análisis. Todo el mundo relacionado con el subconsciente, así como el llamado conocimiento de sentido común, escapa habitualmente al esfuerzo individual de reflexión.

La entrevista grupal tiene lugar para facilitar la salida a flote de todo este mundo. El grupo potencia este trabajo del entrevistador individual creando una situación en la que se multiplican las reacciones individuales y se intercambian más puntos de vista, con lo que los sujetos encuentran una mayor facilidad y estímulo para descubrir, analizar y describir sus propias ideas, sus actitudes, sus experiencias y su conducta. Bombardeados y provocados por la discusión del grupo ganan en profundidad, en riqueza y en rapidez captadora y expresiva. (...)

En segundo lugar, y al margen del contexto decisional en el que resulta un enriquecimiento provocador, el grupo crea un contexto social en el que los datos, las afirmaciones, las observaciones, adquieren un sentido más real y manifiesto del que pueden obtener en el contexto individual de la relación personal entrevistadorentrevistado. (...)

En tercer lugar, el grupo ofrece la oportunidad de entrevistar a más sujetos en menos tiempo, con el consiguiente ahorro de dinero, de energía y de tiempo... en igualdad de circunstancias, se conoce más y mejor un problema no individual, a través de una entrevista grupal.”(Ruiz e Ispizua, 1989:161-162)

Los sujetos que formaron el grupo entrevistado fueron elegidos como expertos dentro del universo de sujetos relacionados con el tema de análisis (Ruiz e Ispizua, 1989:160). En el caso de la entrevista de funcionarios académicos, se consideró la relación jerárquica entre ellos y por eso se dividieron en dos grupos. Por un lado se hizo entrevista grupal a funcionarios sin rango jerárquico y por otro a dos autoridades de la universidad. Los denominados funcionarios contaban entre sí con una relación a lo más de coordinación de labores de trabajo. El grupo denominado autoridades abarcó a partir del nivel jerárquico que implicaba relación de mando y por eso a partir del Director de la Escuela se consideró como nivel de autoridad. El motivo principal para separar estos dos grupos de funcionarios fue para disminuir situaciones comprometedoras, "...de forma que nadie se vea cohibido en su respuesta por miedo a cometer una indiscreción, revelar un secreto, ser imprudente al exponer su propia intimidad”. (Ruiz e Ispizua, 1989:166)

\subsubsection{Entrevista a los estudiantes}

Se seleccionaron estudiantes de último nivel dentro de la universidad. Lo anterior con el objetivo de tener un estudiante experimentado dentro del proceso educativo a distancia, pero que tuviera aun relación con el sistema. Específicamente se escogió al grupo del curso "Tendencias futuras" matriculado para el segundo cuatrimestre de 1997. Por otra parte, como el estudio se basa en un 
enfoque sobre las destrezas que debe tener el estudiante que sale de la universidad, la valoración de las mismas es más contundente si la realiza un estudiante próximo a graduarse, a uno aun inmerso en el proceso educativo y por lo tanto en proceso de adquirir destrezas.

La entrevista grupal se realizó en una única sesión dividida en dos secciones: La primera duró hora y media y consistió en una sesión basada en un guión esquemático, que orientó la conversación de lo general a lo particular de los temas (Ruiz e Ispizua, 1989: 164). Se les ofreció un esquema sobre lo que la sociedad necesita de ellos y se les solicitó que expresaran si se sentían o no preparados para enfrentar esos retos. Se fueron analizando punto por punto los conocimientos y destrezas que la sociedad plantea y se le dio espacio para que expresaran si estaban o no de acuerdo con ellas.

La segunda sección duró media hora, y consistió en recoger las impresiones que provocaban en los estudiantes la presentación de un "Pensamiento" referido a lo que teóricamente se plantea que es la educación a distancia. Esta parte de la entrevista en grupo además de enfrentar lo real, la experiencia vivida, lo cotidiano de la vida estudiantil con lo que el sistema a distancia dice que es y desea ofrecer, permitió hacer un control sobre las manifestaciones externadas en la primera sección de la entrevista.

\subsubsection{Entrevista a los empresarios}

Un agente muy importante en la contextualización de la realidad de las carreras de administración es el sector que contrata los servicios de los profesionales que prepara la universidad. Por este motivo se acudió a un grupo de empresarios para conocer sus opiniones acerca del perfil de graduado que necesita el círculo en que ellos están inmersos.

Por lo anterior, las opiniones externadas por los empresarios responden a una visión general acerca del graduado universitario, independientemente de la institución que le otorga el título.

Se seleccionaron a integrantes de diferentes tipos de empresas y por ello se contó con los siguientes representantes:

Uu representante de la Cámara de Industrias.

uedianos y pequeños empresarios líderes y de reconocido éxito en el respectivo sector en donde se encuentran inmersos pero que además con una visión global de dicho sector y de la economía nacional.

urofesionales que ejercen liberalmente sus profesiones y que cuentan con especialidades afines a las que imparte la UNED. Estos de reconocida solidez académica, de éxito en sus empresas consultoras y/o que tuvieran experiencia académica de alto nivel.

La entrevista grupal a estos informantes estuvo dividida en tres partes: La primera estuvo basada en generación de ideas con base en un guión esquemático sobre lo que sus empresas requerían de un graduado en administración de empresas.

En la segunda parte se buscó provocar una reacción por parte de ellos, para lo cual se les entregó primero un esquema gráfico sobre lo que se puede considerar como GERENTE MODERNO y luego una hoja resumen que caracterizaba una EMPRESA MODERNA, de acuerdo a la concepción teórica de una sociedad moderna planteada anteriormente. Esto por cuanto no siempre el tipo de empresas que existen en estas sociedades se ajustan al modelo de empresa moderna como la planteada aquí, y posteriormente se escucharon las reacciones sobre el particular. 
Esta sección pretendía validar los perfiles de tipo de "gerente" y "empresa" con que se ha venido trabajando y a partir de la cual se definieron las destrezas y conocimientos en el punto anterior.

Es importante aclarar, como lo señala Escotet (1992), que como la universidad debe situarse adelante de los cambios y no detrás de ellos, el modelo de sociedad, de empresa y de gerente que se plantea, no es necesariamente el que actualmente se encuentra desarrollado en el país. Por el contrario, el programa académico que de aquí surge debe adelantarse a las necesidades actuales del sector y más bien orientados hacia los cambios que la tecnología irremediablemente provoca.

Para trabajar en estas entrevistas se procedió con la misma metodología de transcripción y análisis realizado con los estudiantes.

\subsubsection{Entrevista a los funcionarios académicos}

Para la entrevista grupal de los funcionarios académicos, se dividió en dos la sesión de trabajo. La primera, al igual que en el caso anterior, consistió en una generación de ideas, y la segunda en la valoración del resumen de una empresa moderna y un gerente moderno.

En la segunda parte de la entrevista se buscó la reacción de los académicos ante opiniones vertidas por los estudiantes y empresarios sobre el sistema a distancia, la calidad académica, aspectos pedagógicos, las destrezas de un graduado y otros aspectos.

\subsubsection{Entrevista individual}

Por los motivos arriba expuestos, en forma individual se entrevistó al Vicerrector Académico y al Director de la Escuela.

Con el señor Vicerrector Académico la entrevista se dirigió a profundizar los aspectos que las autoridades consideran sobre la temática de la investigación. Con respecto al Director de la Escuela, se siguió la misma guía utilizada en la entrevista grupal de los funcionarios académicos.

Los resultados de estas entrevistas se presentan adelante en el cuadro 1.

\subsection{APLICACIÓN DE LOS CRITERIOS DE CALIDAD}

De acuerdo con los criterios de L'Ecuyer (1997) descritos, se analizaron los contenidos del programa frente a las necesidades planteadas por el modelo teórico de sociedad y por los resultados de las sesiones grupales.

Los resultados fueron los siguientes:

\subsubsection{Pertinencia del programa}

Para que los programas académicos estén acordes con las expectativas y necesidades del mercado actual de trabajo, en parte justificado por las transformaciones que ha sufrido el mercado costarricense, los diferentes grupos, reafirman la necesidad de desarrollar las siguientes destrezas:

u Conocimientos actualizados

u Desarrollo de la creatividad

uspíritu de competencia

u Formación generalista, debe saber tanto de mercadeo, como de finanzas y contabilidad, especialmente importante para la microempresa característico del mercado costarricense, 
aunque con claro dominio de la especialidad.

u Capacidad para plantear y resolver problemas

u Capacidad de dirección y definición de estrategias

u Desarrollo del sentido común

Si observamos el modelo teórico de mercado laboral, estos aspectos están acordes con la teoría administrativa moderna.

Sin embargo, con respecto al trabajo realizado en la Escuela de Administración de la UNED, se observó que los objetivos de los programas de la Escuela de Administración, actualmente establecidos, no están acordes a las expectativas y necesidades del actual mercado de trabajo, (ni de los empresarios, ni del modelo teórico laboral), lo cual parece razonable si se observa que estos fueron redactados a finales de los años 80s, época a partir de la cual el mercado costarricense ha sufrido profundas transformaciones.

Los objetivos planteados para la carrera pretenden:

$\omega$ que el graduado ejecute normas y procedimientos

$u$ que el graduado domine el proceso administrativo, a saber: planear, dirigir, organizar, supervisar y controlar.

$u$ que el graduado establezca estrategias y políticas

Este es el fundamento por el cual se hace la apreciación general de que tanto los objetivos como los contenidos del programa de estudios de la Escuela de Ciencias de la Administración no están acordes con las expectativas y las necesidades del mercado del trabajo que actualmente imperan.

Un análisis profundo de lo expresado tanto por empresarios como por estudiantes, pues existe coincidencia en algunos aspectos entre lo expresado por unos y otros, ofrece el siguiente resultado:

En primer lugar, ambos anotan la debilidad en el conocimiento adquirido. Mas aún, los estudiantes hicieron gran énfasis en el escaso dominio de su especialidad, a pesar de que en muchos casos se tratan gran número de temas, pero se profundiza poco.

En segundo lugar ambos expresan como debilidad el escaso vínculo entre universidad y empresa. Si se estudia la forma en que se está impartiendo la docencia en la UNED, no se puede asegurar que todos los graduados de licenciatura conocen una planta de producción.

En tercer lugar ambos expresan que existen dificultades del graduado para realizar una investigación, y como consecuencia tampoco existe desarrollo adecuado del pensamiento crítico. Si bien este parece ser un problema general de los graduados de administración en todas las universidades, la UNED fomenta esta tendencia ya que su sistema de enseñanza se basa en la apropiación de conocimientos. A ello también debe agregarse que existen problemas para realizar investigaciones, debido a los limitados recursos con que cuenta la biblioteca. Cabe agregar las dificultades todavía mayores para estudiantes que viven en zonas alejadas del área metropolitana y finalmente a la carencia de proyectos de investigación por parte de los profesores.

En cuarto lugar, un sentimiento común es la carencia en cuanto al dominio de otro idioma, sobre todo el inglés, requisito indispensable en el mundo en que vivimos. 
Existen otros criterios que fueron expresados únicamente por los empresarios por cuanto son ellos quienes viven día a día las dificultades al contratar profesionales. Dentro de estos tenemos: el temor del profesional a sumir riesgos, la dificultad para trabajar en equipo, la dificultad para identificar los problemas presentes en el clima organizacional, la falta de actualización en cuanto a los conocimientos, la escasa iniciativa para resolver problemas, poco manejo de paquetes de computación, las dificultades para exponer y defender proyectos.

A pesar de lo anterior, es importante hacer notar que el programa cuenta con algunos de los criterios expresados. Así por ejemplo, los empresarios sostienen que el administrador debe ser un generalista, saber tanto de mercadeo, como de finanzas y contabilidad, opinión que parece razonable sobre todo si observamos que las empresas costarricenses son pequeñas, lo cual hace poco práctico la excesiva especialización. Pero debe abonarse en su favor que el programa actual de la UNED cuenta con esta visión, ya que es un programa generalista con una especialización en las distintas áreas.

\subsubsection{Coherencia del programa}

Un aspecto que demanda atención es el mantenimiento de la continuidad en el tiempo en cuanto a los conocimientos impartidos, de manera que se siga una lógica de exposición en los temas relacionados, donde se evite la discontinuidad para no dificultar la comprensión y reafirmación de éstos.

Aun en procesos de reestructuración de los programas académicos, la coherencia integral debe ser cuidada de manera que no produzca truncamiento que afecte la integración de los conocimientos, y con ello la posibilidad de su aplicación.

Si se analizan los argumentos de los estudiantes, podemos observar que existe discontinuidad en el tiempo en cuanto a los conocimientos que reciben. Es decir, aprueban un curso y mucho tiempo después, en algunos casos años después, reciben el curso siguiente, materias que con frecuencia han sido modificadas por lo que enfrentan serios problemas en el segundo curso ya que se les solicita conocimientos previos que muchas veces no poseen. Las causas de esta observación de los estudiantes puede obedecer a que en la gran mayoría de los casos, los estudiantes tardan 7.4 años y más para obtener su título, según el "Informe de labores del Rector 1986-1996" (UNED, 1996:79), por lo que durante todo ese tiempo van a enfrentar varios cambios de programas. Por otra parte, la flexibilidad con que el estudiante de la UNED puede desarrollar su plan de estudios le permite llevar las asignaturas cuando lo desee sin preocuparse ni por los requisitos ni por el tiempo. Es decir, las verdaderas causas de esa discontinuidad que el estudiante reclama parecen obedecer a la flexibilidad y a la ausencia de requisitos.

\subsubsection{Valor de los métodos pedagógicos y la orientación a los estudiantes}

Para su desenvolvimiento profesional los académicos y los estudiantes requieren estar al día con la realidad empresarial del país y del mundo, ésta se logra realizando proyectos en empresas nacionales y desarrollando estudio de casos, con cierto grado de dificultad, similares a los que la empresa enfrenta en la realidad. Las actividades a realizar en las sesiones de tutorías tienen que ser dinámicas, donde se discuta problemas y soluciones sacados de la empresa costarricense.

El conocimiento de los nuevos paradigmas en la Administración, deben ser temas tratados y criticados en los cursos, esto compromete a la universidad y a profesores a disponer de una capacitación permanente que se convierta en beneficios en la formación del estudiante. Pretender cubrir una gran 
cantidad de materias en una solo sesión de tutoría dificulta su asimilación con repercusiones en la formación profesional.

En este sentido es necesario mencionar los criterios expresados, tanto por los académicos entrevistados, como por los estudiantes. Ambos manifiestan que existe un desconocimiento por parte de los estudiantes de la realidad empresarial del país. La educación se limita a desarrollar unos pocos casos en clase y muy simplificados que no reflejan la realidad del mercado laboral. Por otra parte en el caso muy particular de la UNED, los estudiantes citan que las tutorías "no son dinámicas, por el contrario, son muy pasivas", lo cual hace prácticamente imposible la discusión sobre la realidad del país.

Otro de los problemas anotados tanto por académicos como por empresarios y estudiantes es que la gran cantidad de materia asignada para un curso dificulta su asimilación y profundización. Esto es importante en la UNED por cuanto esta Universidad redujo el tiempo de estudio semestral para cada curso al adoptar la modalidad de cuatrimestres.

Finalmente los estudiantes se refieren al aprendizaje memorístico que exige la UNED, lo cual les limita su capacidad de crítica.

\subsubsection{Adecuación de los recursos humanos, materiales y financieros}

El material bibliográfico, el acceso a los laboratorios, la facilidad de exploración en la Red Internet, las charlas de expertos sobre temas especiales, la disponibilidad de material complementario a los libros del mercado y una debida asignación de la carga académica de los profesores que permita la actualización de conocimientos y la investigación son factores a considerar para dotar de los recursos que exige un programa académico.

Como ya se ha mencionado anteriormente, la escasez de material bibliográfico en las bibliotecas, la dificultad de acceso a laboratorios, a la red Internet, a charlas de expertos, la carencia de material complementario a los libros de mercado y muy especialmente el exceso de carga académica de los profesores en labores docentes, les dificulta la actualización y la investigación. Son todos estos factores que conspiran para que la Escuela pueda contar con recursos humanos adecuados.

\subsubsection{Eficacia del programa}

Diseñar un programa académico para un sistema educativo a distancia de manera tal que ofrezca garantía de realización a los estudiantes que superan un proceso de selección previamente establecido, es parte de los aspectos que se deben cuidar. Por un lado, está la necesidad de definir mecanismos de selección que den garantía de éxito acorde con la exigencia del programa, y por otro, permitir la flexibilidad inherente a los sistemas a distancia, sin provocar frustración por imposibilidad de incumplimiento del programa, que se traduzca en bajo rendimiento y deserción.

Es de suma importancia resaltar en este aspecto que son los mismos estudiantes quienes expresan que el programa no es muy selectivo en la escogencia de los estudiantes, y de acuerdo a su criterio no todos los estudiantes cuentan con los sanos deseos de aprender, sino que en algunos casos se viene a obtener un título solamente. Por lo tanto, en criterio de algunos estudiantes, la Universidad debería detectar estos casos y ser más selectiva en la admisión de los mismos.

Si se analizan los objetivos establecidos para los programas de la Escuela de Administración, podemos observar cómo éstos plantean un graduado al nivel de licenciatura con capacidad para establecer 
estrategias y políticas. Sin embargo, dado que el sistema de enseñanza de la UNED, se limita a apropiar conocimientos y con algunas excepciones se envía al estudiante a realizar pequeños estudios de campo, puede deducirse entonces, que con tal metodología, a lo sumo se alcanzará el primer objetivo, a saber: ejecutar normas y procedimientos operativos, pero no así la capacidad para integrar conocimientos ni la formación de líderes capaces de dirigir y establecer estrategias.

\subsubsection{Calidad de la gestión del programa}

La agilidad del proceso administrativo a que debe ser expuesto el estudiante, especialmente los canales de comunicación disponibles para los servicios que éste demanda, deben generar satisfacción por su accesibilidad en tiempo, espacio y confiabilidad. Lo anterior es importante porque en una universidad a distancia se requiere establecer mucha comunicación por vías formales entre profesores y alumnos, vías que generalmente en la UNED cuentan con grandes distorsiones, puesto que existe poca tecnología de apoyo en este sentido y se cuenta únicamente con un correo electrónico que no es muy expedito.

En el cuadro que se plantea a continuación se resumen los problemas y sus causas, según el resultado de las entrevistas realizadas, las cuales han sido clasificadas de acuerdo a los criterios de calidad del modelo aquí planteado.

Cuadro 1: Problemas en la formación de los estudiantes según criterios de calidad

\begin{tabular}{|c|c|c|c|c|}
\hline $\begin{array}{l}\text { CRITERIO } \\
\text { DE } \\
\text { CALIDAD }\end{array}$ & PROBLEMA & \begin{tabular}{|c|} 
DESTRE \\
- \\
ZAS \\
AFECTA \\
- \\
DAS (1) \\
\end{tabular} & $\begin{array}{l}\text { A } \\
\text { JUICIO } \\
\text { DE: }\end{array}$ & CAUSAS \\
\hline \multirow[t]{3}{*}{$\begin{array}{l}\text { La } \\
\text { pertinencia } \\
\text { del } \\
\text { programa }\end{array}$} & $\begin{array}{l}\text { Debilidad en el conocimiento } \\
\text { básico de todas las áreas de la } \\
\text { Administración. Ausencia de una } \\
\text { formación generalista. El } \\
\text { profesional debe saber de todas } \\
\text { las áreas (mercadeo, finanzas, } \\
\text { etc.) }\end{array}$ & 1 & $\begin{array}{l}\text { Estudiant } \\
\text { es } \\
\text { Empresari } \\
\text { os }\end{array}$ & $\begin{array}{l}\text { Cursos de gran generalidad sin } \\
\text { profundizar en temas } \\
\text { específicos }\end{array}$ \\
\hline & $\begin{array}{l}\text { Debilidades en el dominio de la } \\
\text { especialidad. } \\
\text { Poca profundidad en los temas } \\
\text { específicos del énfasis }\end{array}$ & 1 & $\begin{array}{l}\text { Estudiant } \\
\text { es }\end{array}$ & $\begin{array}{l}\text { No se hacen prácticas que } \\
\text { permitan generar las } \\
\text { experiencias del aprendizaje }\end{array}$ \\
\hline & $\begin{array}{l}\text { Temores a la competencia, se } \\
\text { debe poder asumir riesgos, saber } \\
\text { establecer alianzas, etc. }\end{array}$ & 6 & $\begin{array}{l}\text { Empresari } \\
\text { os }\end{array}$ & $\begin{array}{l}\text { No existen dinámicas que } \\
\text { permitan al estudiante } \\
\text { enfrentarse o estudiar } \\
\text { situaciones con situaciones } \\
\text { similares a las que se encontrará } \\
\text { en el mercado laboral }\end{array}$ \\
\hline
\end{tabular}




\begin{tabular}{|c|c|c|c|}
\hline $\begin{array}{l}\text { Dificultad de trabajo en equipo, } \\
\text { poca habilidad de manejo de } \\
\text { grupo, en integrar equipos de } \\
\text { trabajo, saber contratar } \\
\text { trabajadores y falta humanidad en } \\
\text { la dirección de personal } \\
\end{array}$ & $\begin{array}{l}3 \\
4\end{array}$ & $\begin{array}{l}\text { Empresari } \\
\text { os }\end{array}$ & $\begin{array}{l}\text { Por la modalidad de estudio, no } \\
\text { se permite este tipo de práctica. }\end{array}$ \\
\hline Fallas en el manejo de personal & 4 & $\begin{array}{l}\text { Empresari } \\
\text { os }\end{array}$ & $\begin{array}{l}\text { Se estudian casos muy simples } \\
\text { y no se hacen prácticas en la } \\
\text { realidad. }\end{array}$ \\
\hline $\begin{array}{l}\text { Dificultad en identificar la } \\
\text { identidad de la empresa y el } \\
\text { clima institucional }\end{array}$ & 8 & $\begin{array}{l}\text { Empresari } \\
\text { os }\end{array}$ & $\begin{array}{l}\text { No se brindan enfoques } \\
\text { prácticos. }\end{array}$ \\
\hline $\begin{array}{l}\text { Dificultad en mantenerse } \\
\text { actualizado }\end{array}$ & 1 & $\begin{array}{l}\text { Empresari } \\
\text { os }\end{array}$ & $\begin{array}{l}\text { Carencia de un programa de } \\
\text { post-grado de alta calidad a } \\
\text { profesores. } \\
\text { Dificultad de acceso a } \\
\text { bibliotecas actualizadas. } \\
\end{array}$ \\
\hline $\begin{array}{l}\text { Debilidades en el vínculo } \\
\text { universidad-empresa }\end{array}$ & 8 & \begin{tabular}{|l|} 
Empresari \\
os \\
Académic \\
os \\
Estudiant \\
es \\
\end{tabular} & $\begin{array}{l}\text { La UNED no tiene vínculo con } \\
\text { las empresas que permitan al } \\
\text { estudiante realizar prácticas. }\end{array}$ \\
\hline $\begin{array}{l}\text { Dificultad en la relación con el } \\
\text { mundo acorde con las exigencias } \\
\text { de la globalización }\end{array}$ & 1 & $\begin{array}{l}\text { Empresari } \\
\text { os }\end{array}$ & $\begin{array}{l}\text { No se cuenta con laboratorios } \\
\text { de idiomas, poco acceso a } \\
\text { Internet, poco acceso a estudios } \\
\text { de mercados Internacionales, } \\
\text { ningún intercambio con otras } \\
\text { universidades o instituciones. } \\
\end{array}$ \\
\hline \begin{tabular}{|l|} 
Debilidades en las relaciones \\
interpersonales
\end{tabular} & $3-4$ & \begin{tabular}{|l|}
$\begin{array}{l}\text { Empresari } \\
\text { os }\end{array}$ \\
\end{tabular} & $\begin{array}{l}\text { Poca tecnología que permita } \\
\text { relaciones interpersonales }\end{array}$ \\
\hline $\begin{array}{l}\begin{array}{l}\text { Poca disposición al aprendizaje } \\
\text { permanente }\end{array} \\
\end{array}$ & 1 & \begin{tabular}{|l|} 
Empresari \\
os
\end{tabular} & $\begin{array}{l}\text { Existen pocos trabajos de } \\
\text { investigación bibliográfica. }\end{array}$ \\
\hline $\begin{array}{l}\text { Problemas en aplicación de la } \\
\text { teoría }\end{array}$ & 6 & \begin{tabular}{|l|} 
Empresari \\
os \\
Estudiant \\
es
\end{tabular} & Falta de prácticas. \\
\hline $\begin{array}{l}\text { Poca habilidad para dirigir con } \\
\text { los recursos disponibles }\end{array}$ & $4-2$ & \begin{tabular}{|l|} 
Estudiant \\
es \\
Empresari \\
os
\end{tabular} & Falta de enfoques prácticos. \\
\hline $\begin{array}{l}\text { Poca iniciativa para acceder a } \\
\text { buenas fuentes de información }\end{array}$ & 2 & $\begin{array}{l}\text { Empresari } \\
\text { os }\end{array}$ & $\begin{array}{l}\text { Carencia de acceso a } \\
\text { bibliotecas. }\end{array}$ \\
\hline $\begin{array}{l}\text { Dificultad para encontrar canales } \\
\text { que generen utilidades a las } \\
\text { empresas }\end{array}$ & 8 & $\begin{array}{l}\text { Empresari } \\
\text { os }\end{array}$ & $\begin{array}{l}\text { Falta de enfoques prácticos que } \\
\text { permitan desarrollar su } \\
\text { creatividad. }\end{array}$ \\
\hline
\end{tabular}




\begin{tabular}{|c|c|c|c|c|}
\hline & $\begin{array}{l}\text { Poco desarrollo del sentido } \\
\text { común para resolver problemas }\end{array}$ & 2 & $\begin{array}{l}\text { Empresari } \\
\text { os } \\
\text { Estudiant } \\
\text { es } \\
\end{array}$ & \\
\hline & \begin{tabular}{|l} 
Poca capacidad para innovar \\
\end{tabular} & 2 & $\begin{array}{l}\text { Empresari } \\
\text { os }\end{array}$ & \\
\hline & $\begin{array}{l}\text { Poca habilidad para hacer análisis } \\
\text { financieros y contables }\end{array}$ & 2 & $\begin{array}{l}\text { Empresari } \\
\text { os }\end{array}$ & \\
\hline & $\begin{array}{l}\text { Dificultad en la administración } \\
\text { del tiempo }\end{array}$ & 2 & $\begin{array}{l}\text { Empresari } \\
\text { os }\end{array}$ & \\
\hline & \begin{tabular}{|l|} 
Desactualización de los \\
contenidos de los programas pese \\
ser una profesión de cambio \\
constante
\end{tabular} & 1 & $\begin{array}{l}\text { Académic } \\
\text { os }\end{array}$ & $\begin{array}{l}\text { Rigidez en introducción de } \\
\text { cambios del material didáctico } \\
\text { Política de optimización de } \\
\text { existencias en el inventario de } \\
\text { libros } \\
\quad \text { La duración del proceso de } \\
\text { creación de una carrera es } \\
\text { demasiado lento } \\
\end{array}$ \\
\hline & $\begin{array}{l}\text { Poco manejo de paquetes } \\
\text { computacionales }\end{array}$ & 7 & $\begin{array}{l}\text { Empresari } \\
\text { os }\end{array}$ & Falta de tecnología. \\
\hline & $\begin{array}{l}\text { El estudiante no sabe investigar y } \\
\text { los académicos tampoco } \\
\text { investigan }\end{array}$ & 8 & $\begin{array}{l}\text { Académic } \\
\text { os } \\
\text { Estudiant } \\
\text { e }\end{array}$ & $\begin{array}{l}\text { Escasez de material } \\
\text { bibliográfico en las bibliotecas } \\
\text { de la UNED. } \\
\text { Dificultad acceso Red Internet } \\
\end{array}$ \\
\hline & $\begin{array}{l}\text { Problemas de manejo del idioma } \\
\text { inglés básico para la } \\
\text { actualización en una disciplina } \\
\text { que cambia constantemente }\end{array}$ & 11 & $\begin{array}{l}\text { Empresari } \\
\text { os } \\
\text { Académic } \\
\text { os }\end{array}$ & $\begin{array}{l}\text { Carencia de laboratorios o } \\
\text { convenios con otras } \\
\text { instituciones }\end{array}$ \\
\hline & $\begin{array}{l}\text { Los gerentes deben generar } \\
\text { confianza y respeto, y la } \\
\text { formación académica no da esa } \\
\text { condición } \\
\end{array}$ & 6 & $\begin{array}{l}\text { Empresari } \\
\text { os }\end{array}$ & $\begin{array}{l}\text { Faltan actividades académicas } \\
\text { que faciliten esa condición }\end{array}$ \\
\hline & $\begin{array}{l}\text { No hay desarrollo de } \\
\text { pensamiento crítico }\end{array}$ & 8 & $\begin{array}{l}\text { Empresari } \\
\text { os } \\
\text { Estudiant } \\
\text { es }\end{array}$ & \\
\hline & \begin{tabular}{|l|} 
No se tiene capacidad para \\
exponer y defender proyectos
\end{tabular} & 9 & $\begin{array}{l}\text { Estudiant } \\
\text { es }\end{array}$ & \\
\hline $\begin{array}{l}\text { La } \\
\text { coherencia } \\
\text { del } \\
\text { programa }\end{array}$ & $\begin{array}{l}\text { La deflactación del programa } \\
\text { académico se realizó sin } \\
\text { considerar a los docentes } \\
\text { responsables, se perdió el } \\
\text { enfoque carrera-céntrico, } \\
\text { alterando la coherencia del } \\
\text { programa } \\
\end{array}$ & & $\begin{array}{l}\text { Académic } \\
\text { os } \\
\text { Estudiant } \\
\text { es }\end{array}$ & $\begin{array}{l}\text { Poca capacidad de trabajo en } \\
\text { equipo. }\end{array}$ \\
\hline & Discontinuidad del conocimiento & & $\begin{array}{l}\text { Estudiant } \\
\text { es }\end{array}$ & \\
\hline
\end{tabular}




\begin{tabular}{|c|c|c|c|c|}
\hline \multirow{8}{*}{$\begin{array}{l}\text { El valor de } \\
\text { los métodos } \\
\text { pedagógicos } \\
\text { y la } \\
\text { orientación } \\
\text { a los } \\
\text { estudiantes }\end{array}$} & Distanciamiento en el tiempo & & $\begin{array}{l}\text { Estudiant } \\
\text { es }\end{array}$ & \\
\hline & Ausencia de requisitos & & $\begin{array}{l}\text { Estudiant } \\
\text { es }\end{array}$ & \\
\hline & $\begin{array}{l}\text { Gran cantidad de materia en un } \\
\text { curso dificulta su asimilación, no } \\
\text { se puede profundizar y en las } \\
\text { tutorías difícilmente se atienden } \\
\text { todas las necesidades }\end{array}$ & 1 & $\begin{array}{l}\text { Estudiant } \\
\text { es } \\
\text { Empresari } \\
\text { os } \\
\text { Académic } \\
\text { os }\end{array}$ & $\begin{array}{l}\text { Enfoques muy generales con } \\
\text { mucho contenido y poca } \\
\text { profundización en aspectos } \\
\text { específicos. }\end{array}$ \\
\hline & $\begin{array}{l}\text { Desconocimiento por parte del } \\
\text { estudiante de la realidad } \\
\text { empresarial del país }\end{array}$ & & $\begin{array}{l}\text { Académic } \\
\text { os } \\
\text { Estudiant } \\
\text { es }\end{array}$ & Carencia de enfoques prácticos \\
\hline & $\begin{array}{l}\text { Se desarrollan pocos casos en } \\
\text { clase y muy simplificados que no } \\
\text { reflejan la realidad del mercado } \\
\text { laboral }\end{array}$ & & $\begin{array}{l}\text { Académic } \\
\text { os } \\
\text { Estudiant } \\
\text { es }\end{array}$ & $\begin{array}{l}\text { Hay pocas prácticas en las } \\
\text { empresas y en algunos casos no } \\
\text { existen. }\end{array}$ \\
\hline & \begin{tabular}{|l|} 
Las tutorías no son dinámicas, \\
por el contrario muy pasivas
\end{tabular} & & $\begin{array}{l}\text { Estudiant } \\
\text { es }\end{array}$ & $\begin{array}{l}\text { Ausencias de tecnología o } \\
\text { cambios en el modelo tutorial }\end{array}$ \\
\hline & Respeto a los estudiantes & & $\begin{array}{l}\text { Estudiant } \\
\text { es }\end{array}$ & \\
\hline & Aprendizaje memorístico & & $\begin{array}{l}\text { Estudiant } \\
\text { es }\end{array}$ & \\
\hline \multirow{4}{*}{\begin{tabular}{|l} 
La \\
adecuación \\
de los \\
recursos \\
humanos, \\
materiales y \\
financieros
\end{tabular}} & $\begin{array}{l}\text { Escasez de material bibliográfico } \\
\text { en las bibliotecas de la UNED }\end{array}$ & 1 & $\begin{array}{l}\text { Académic } \\
\text { os } \\
\text { Estudiant } \\
\text { es } \\
\end{array}$ & \\
\hline & $\begin{array}{l}\text { Exceso de carga académica a los } \\
\text { profesores que los dificulta } \\
\text { actualizarse e investigar } \\
\end{array}$ & & $\begin{array}{l}\text { Académic } \\
\text { os }\end{array}$ & \\
\hline & \begin{tabular}{|l} 
Dificultad de acceso a \\
laboratorios, a la Red Internet, a \\
charlas de expertos que solo se \\
ofrecen en San José y aumenta la \\
inequidad de la educación \\
\end{tabular} & 8 & $\begin{array}{l}\text { Académic } \\
\text { os } \\
\text { Estudiant } \\
\text { es }\end{array}$ & Poca tecnología \\
\hline & $\begin{array}{l}\text { Cuando se emplean libros del } \\
\text { mercado no se tienen guías } \\
\text { académicas que facilite el } \\
\text { aprendizaje independiente y } \\
\text { adapte ejemplos adecuados a } \\
\text { nuestro medio. } \\
\end{array}$ & & $\begin{array}{l}\text { Académic } \\
\text { os } \\
\text { Estudiant } \\
\text { es }\end{array}$ & \\
\hline $\begin{array}{l}\text { La eficacia } \\
\text { del } \\
\text { programa }\end{array}$ & $\begin{array}{l}\text { No se es muy selectivo en la } \\
\text { escogencia de los estudiantes }\end{array}$ & & $\begin{array}{l}\text { Estudiant } \\
\text { es }\end{array}$ & \\
\hline La calidad & \begin{tabular}{|l|} 
Disponer de una central \\
telefónica con correo de vos
\end{tabular} & & & \\
\hline
\end{tabular}




\begin{tabular}{|l|l|l|l|l|}
\hline $\begin{array}{l}\text { de la gestión } \\
\text { del } \\
\text { programa }\end{array}$ & $\begin{array}{l}\text { Los mensajes dejados a los } \\
\text { académicos en una mayoría no } \\
\text { reciben respuesta. }\end{array}$ & $\begin{array}{l}\text { Estudiant } \\
\text { es }\end{array}$ & \\
\cline { 2 - 4 } & $\begin{array}{l}\text { Problemas en el pago oportuno a } \\
\text { los profesores genera } \\
\text { desmotivación tanto en } \\
\text { profesores como en estudiantes }\end{array}$ & & $\begin{array}{l}\text { Estudiant } \\
\text { es }\end{array}$ & \\
\cline { 2 - 5 } & $\begin{array}{l}\text { Desórdenes administrativos. } \\
\text { Improvisación. }\end{array}$ & & $\begin{array}{l}\text { Estudiant } \\
\text { es }\end{array}$ & \\
\hline
\end{tabular}

(1) Los números indicados en esta columna corresponden a los asignados a las destrezas en el cuadro \# 2.

\subsection{ESQUEMA DE LAS DESTREZAS}

Como resultado de la aplicación de este modelo, se obtuvo el siguiente cuadro, el cual esquematiza para cada destreza el tipo de conocimiento y actividades que se requieren para adquirirla, así como las asignaturas que existen actualmente y que deberían generar la destreza descrita.

Aquí se plantea la propuesta final del modelo teórico de mercado laboral que se ofreció al inicio de esta sección.

Cuadro 2: Destrezas, conocimientos, habilidades y los cursos correspondientes de la carrera de Administración de la UNED

\begin{tabular}{|c|c|c|c|}
\hline DESTREZAS & CONOCIMIENTO & ACTIVIDADES & ASIGNATURAS \\
\hline $\begin{array}{l}\text { 1) } \\
\text { - Visión amplia del } \\
\text { mundo: } \\
\text { * Actualizarse } \\
\text { permanentemente } \\
\text { * Dominio de la } \\
\text { especialidad y } \\
\text { conocimiento general } \\
\text { de todas las áreas }\end{array}$ & $\begin{array}{l}\text { - Generales en: } \\
\text { * Cambio tecnológico } \\
\text { * Economía (enfoque } \\
\text { global) } \\
\text { * Política } \\
\text { * Historia (Social, } \\
\text { Educativo, } \\
\text { Demográfico) } \\
\text { - Necesidad de } \\
\text { actualización } \\
\text { permanente ante el } \\
\text { cambio constante. } \\
\text { - Idioma }\end{array}$ & $\begin{array}{l}\text { - Discusión en grupo } \\
\text { - Investigación } \\
\text { bibliográfica } \\
\text { - Fomentar lectura } \\
\text { - Casos }\end{array}$ & $\begin{array}{l}* \text { Humanidades } \\
* \text { Asignaturas de cada } \\
\text { especialidad }\end{array}$ \\
\hline $\begin{array}{l}\text { 2) } \\
\text { - Disposición al } \\
\text { cambio: }\end{array}$ & $\begin{array}{l}\text { - Problemas Históricos } \\
\text { en cambios de } \\
\text { organización. } \\
\text { - Potencial creativo del } \\
\text { ser humano }\end{array}$ & $\begin{array}{l}\text { - Dinámicas de auto } \\
\text { reflexión y desarrollo } \\
\text { de la personalidad. }\end{array}$ & $\begin{array}{l}* \text { Cambio } \\
\text { organizacional } \\
* \text { Nuevos enfoques en: } \\
\text { Mercadeo }\end{array}$ \\
\hline
\end{tabular}




\begin{tabular}{|c|c|c|c|}
\hline $\begin{array}{l}* \text { Mente abierta } \\
* \text { Desarrollo de la } \\
\text { creatividad } \\
\text { * Autodisciplina y } \\
\text { responsabilidad }\end{array}$ & $\begin{array}{l}\text { - Creatividad para el s. } \\
\text { XXI } \\
\text { - Psicología } \\
\text { - Conocimiento de las } \\
\text { actividades de la } \\
\text { empresa } \\
\text { y las necesidades del } \\
\text { cliente }\end{array}$ & - Casos & $\begin{array}{l}\text { Producción } \\
\text { Administración }\end{array}$ \\
\hline $\begin{array}{l}\text { 3) } \\
\text { Capacidad de trabajo } \\
\text { en } \\
\text { equipo }\end{array}$ & & $\begin{array}{l}\text { Presentación de } \\
\text { proyectos en grupo }\end{array}$ & $\begin{array}{l}\text { * Comportamiento } \\
\text { organizacional } \\
\text { * Aplicación en otras } \\
\text { asignaturas }\end{array}$ \\
\hline $\begin{array}{l}\text { 4) } \\
\text { - Líder: } \\
\text { * Facilitador } \\
\text { * Democrático } \\
\text { * Disposición } \\
\text { participativa }\end{array}$ & $\begin{array}{l}\text { - Líder moderno } \\
\text { - Empresa moderna } \\
\text { - Comportamiento de } \\
\text { un líder }\end{array}$ & $\begin{array}{l}\text { - Aplicaciones } \\
\text { prácticas } \\
\text { - Instrumentos de } \\
\text { autoevaluación }\end{array}$ & $\begin{array}{l}\text { * Administración. } \\
\text { general I-II } \\
\text { * Comportamiento } \\
\text { organizacional. }\end{array}$ \\
\hline $\begin{array}{l}\text { 5) } \\
\text { - Excelencia } \\
\text { - Concepto de calidad } \\
\text { en su quehacer }\end{array}$ & $\begin{array}{l}\text { - Calidad total } \\
\text { - Calidad de } \\
\text { producción } \\
\text { - Calidad servicios }\end{array}$ & $\begin{array}{l}\text { - Reuniones de grupos } \\
\text { de expertos y } \\
\text { discusión } \\
\text { de temas de alto nivel }\end{array}$ & $\begin{array}{l}\text { * Producción } \\
\text { Mercadeo (área de } \\
\text { servicios- calidad de } \\
\text { servicios) }\end{array}$ \\
\hline $\begin{array}{l}\text { 6) } \\
\text { - Disposición al riesgo: } \\
\text { * Seguridad al actuar } \\
\text { * Gran sentido de la } \\
\text { competencia }\end{array}$ & $\begin{array}{l}\text { - Aplicación de } \\
\text { conocimiento general } \\
\text { de la empresa y del } \\
\text { contexto a situaciones } \\
\text { reales o simuladas }\end{array}$ & $\begin{array}{l}\text { - Dinámica de } \\
\text { competencia entre } \\
\text { grupos simulando la } \\
\text { vida real }\end{array}$ & Estrategia empresarial \\
\hline $\begin{array}{l}\text { 7) } \\
\text { - Amplia cultura } \\
\text { informática }\end{array}$ & $\begin{array}{l}\text { - Manejo de \# paquetes } \\
\text { informáticos, bases de } \\
\text { datos y herramientas } \\
\text { estadísticas y } \\
\text { financieras }\end{array}$ & $\begin{array}{l}\text { - Ampliación de los } \\
\text { programas } \\
\text { computacionales en } \\
\text { cursos específicos }\end{array}$ & $\begin{array}{l}\text { * Sistemas de } \\
\text { Información } \\
\text { * Contabilidad } \\
\text { * Estadística } \\
\text { * Finanzas } \\
\end{array}$ \\
\hline
\end{tabular}




\begin{tabular}{|c|c|c|c|}
\hline $\begin{array}{l}\text { 8) } \\
\text { Capacidad de análisis y } \\
\text { síntesis: } \\
\text { * En el entorno laboral } \\
\text { y empresarial } \\
\text { * Sentido pragmático } \\
\text { (sentido común) } \\
\text { * Uso de la } \\
\text { investigación }\end{array}$ & $\begin{array}{l}\text { - Amplio conocimiento } \\
\text { sobre las situaciones } \\
\text { reales de las empresas } \\
\text { y el entorno } \\
\text { - Investigar y resolver } \\
\text { problemas concretos }\end{array}$ & $\begin{array}{l}\text { - Casos } \\
\text { - Investigaciones } \\
\text { - Trabajos en empresas }\end{array}$ & $\begin{array}{l}\text { - Comportamiento } \\
\text { organizacional } \\
\text { - Técnicas de } \\
\text { investigación } \\
\text { - Estrategia empresarial } \\
\text { - Asignaturas de } \\
\text { producción }\end{array}$ \\
\hline $\begin{array}{l}\text { 9) } \\
\text { - Capacidad de } \\
\text { expresión oral y } \\
\text { escrita: } \\
\text { * Negociación } \\
\text { * Convencimiento }\end{array}$ & $\begin{array}{l}\text { - Gramática y } \\
\text { compresión de lectura } \\
\text { aplicados }\end{array}$ & $\begin{array}{l}\text { - Realizar síntesis y } \\
\text { análisis } \\
\text { - Comunicación escrita } \\
\text { ligada a la empresa } \\
\text { - Aprender a hacer un } \\
\text { curriculum, informes } \\
\text { financieros, cartas, } \\
\text { resúmenes ejecutivos. }\end{array}$ & $\begin{array}{l}\text { - Comunicación oral y } \\
\text { escrita }\end{array}$ \\
\hline $\begin{array}{l}\text { 10) } \\
\text { - Concientización en la } \\
\text { protección del medio } \\
\text { ambiente }\end{array}$ & $\begin{array}{l}\text { - Conocer casos de } \\
\text { experiencias reales } \\
\text { - Datos sobre } \\
\text { componentes } \\
\text { químicos y } \\
\text { contaminación } \\
\text { - Ubicar fuentes de } \\
\text { información sobre } \\
\text { medio ambiente }\end{array}$ & $\begin{array}{l}\text { - Trabajos en grupo } \\
\text { sobre investigación }\end{array}$ & $\begin{array}{l}\text { - Producción } \\
\text { - Mantenimiento }\end{array}$ \\
\hline
\end{tabular}

\section{CONCLUSIONES Y RECOMENDACIONES}

La propuesta principal de mejoramiento del programa analizado, ha sido visualizada como una variante educativa en un sistema a distancia por sus características particulares. En la actualidad el paquete instruccional está basado en el medio impreso (medio maestro), tutorías presenciales y telefónica, y ocasionalmente apoyo audiovisual. El apoyo en tecnologías de información está en proceso de experimentación, y no se ha concretado en ningún curso en oferta, esa situación lleva a introducir cierto nivel de presencialidad para así poder suplir la necesidad insoslayable de lograr la comunicación bidireccional profesor-alumno, alumno-alumno, y a su vez, logre generar las destrezas identificadas. Obviamente, conforme el proyecto de tecnología académica se concrete, se podrá sustituir ésta presencialidad por una mediatizada por la tecnología de información, que en esencia busca la calidad de comunicación entre los medios didácticos y el estudiante. 
Tomando en consideración los aspectos señalados en las secciones anteriores, se manifiesta la necesidad de introducir cambios al programa académico en oferta, que subsanen en alguna medida las debilidades en la formación detectados en los profesionales. Con esta visión se presenta una propuesta enmarcada dentro de la perspectiva siguiente:

(u Posibilidad de ser implementado en el corto plazo, y en consecuencia con un enfoque parcial para lograr la oportunidad deseada.

un Enfocado en las áreas sustanciales de su especialidad, en relación directa con un desempeño profesional coherente con la calidad esperada, dado el nivel académico otorgado por la universidad, y acercándose a las expectativas que se tiene a nivel empresarial.

u. Susceptible de modificarse conforme las condiciones tecnológicas se hagan una realidad a nivel de país y al interno de la universidad, pero conservando su esencia en cuanto a la efectividad del enfoque pedagógico identificado, factible de transformarse en una opción de menor presencialidad basada en un desarrollo tecnológico nacional e institucional, ausente en estos momentos.

Evaluada cada cuatrimestre para asegurar la calidad necesaria y permitir la oportunidad de introducir los ajustes a tiempo, con una supervisión a alto nivel.

Por esta razón se propone, además de la propuesta del punto 3.5, la creación de Seminarios de Integración que tienen como propósito generar conocimientos de la realidad empresarial, desarrollar una capacidad crítica, analítica y de síntesis, con una visión práctica ante el constante cambio, así como, afirmar su capacidad para plantear y resolver problemas, con una defensa asertiva de sus soluciones. Serían seminarios que puedan desarrollar las habilidades, destrezas y conocimientos como las planteadas del cuadro No.2.

Lógicamente estos seminarios vienen a solventar necesidades en la forma como la UNED

Para obtener el éxito esperado con los seminarios es requisito indispensable contar con académicos de alto nivel, que disfrute de libertad de cátedra, y sean sometidos a una retroalimentación periódica de sus resultados. Los criterios para considerar el perfil de reclutamiento de esos académicos son los siguientes:

\section{Perfil del docente de los seminarios de integración}

Solidez académica

Capacidad investigativa

Conociientos actualizados

Actitud crítica y analítica

Entusiasta responsable y de buenas relaciones

Conocedor de la realizadad nacional

Receptivo a la crítica

Capacidad de síntesis

Capacidad de dirigir grupos de trabajo

Conocimiento de otros idiomas

Preferiblemente con una especialidad acreditada de una universidad de reconocido prestigio

Los objetivos de estos seminarios serían los siguientes:

wesarrollar una actitud analítica y crítica respecto de las concepciones teóricas existentes en el 
área de especialización.

u Capacidad de integración de conocimientos en la solución práctica de problemas concretos y complejos que normalmente se enfrentan las empresas modernas.

u Capacidad de resumir exponer y defender claramente sus puntos de vista en auditorios competentes.

uesarrollar su capacidad investigativa para la resolución de los problemas propios del campo de especialización.

La modalidad de trabajo de los seminarios implica tener sesiones de encuentro presencial y preferiblemente el desarrollo de trabajo en grupo que involucre aspectos como los siguientes:

Uesarrollo de proyectos en empresas

Visitas a empresa nacionales

u Selección cuidadosa de bibliografía que garantice calidad y actualización cada cuatrimestre

Utilización de recursos audiovisuales disponibles

ux Experimentación con los medios tecnológicos que la universidad vaya adquiriendo, así como el uso de recursos como: servicio Web de la Red Internet, material multimedia, videoconferencia, etc.

De la presente investigación se desprende que existen cursos cuyos requerimientos de aprendizaje están siendo satisfechos al apropiar solo conocimientos, pero se genera un problema para el sistema a distancia, en los cursos donde se requieren la aplicación de conocimientos, o sea, donde se necesita la realización de prácticas, que requieren de un proceso de aprendizaje interactivo. Para enfrentar este problema, en el caso de la educación a distancia se podría lograr por medio de la tecnología interactiva o con la presencialidad. Como solución inmediata se propone la creación de tres seminarios durante la carrera y serían los siguientes:

Seminario básico: Se pretende que en el primer seminario esté orientado a reforzar los conocimientos adquiridos en los dos primeros niveles de la carrera, incluyendo las técnicas de estudio a distancia. Este puede ser un seminario en donde se divida el estudio entre distintas especialidades y lo que se haría es corroborar que el estudiante se encuentre bien preparado hasta ese nivel. Para ello deben definirse estándares sobre lo que se espera que el alumno conozca en esos primeros cursos.

Seminario de especialidad: Para el segundo seminario, se pretende un curso especializado, de manera que se refuerce el énfasis que el estudiante ha seleccionado: finanzas, contabilidad, gestión o cualquier otra. Se pretende que estos sean impartidos por profesores de muy alto nivel dentro de su campo y se impartirá no sólo para reforzar los conocimientos adquiridos hasta ese momento, sino también para ofrecer contenidos muy actualizados.

Estos seminarios deberán además desarrollar destrezas que el alumno necesita en su desempeño profesional como son: trabajo en equipo, análisis, aplicación de modelos a la realidad costarricense, etc. La Universidad deberá contratar, no sólo profesores de alto nivel sino además empresas con funciones relacionadas con cada especialidad, de manera que el alumno pueda integrarse en las prácticas laborales durante algunos días.

Seminario sobre liderazgo: El tercer seminario consistiría en reforzar las destrezas con las que debe contar todo gerente, a saber: resolución de problemas, identificación de estrategias, análisis del entorno, y en general preparación para asumir posiciones de liderazgo. 
La investigación demostró que el desarrollo de destrezas en la carrera de Administración de Empresas, es tan importante como la solidez de los conocimientos adquiridos. Por lo tanto es fundamental crear un modelo pedagógico que oriente por curso las estrategias de aprendizaje, lo cual se trato de realizar como un primer acercamiento en la investigación base del presente documento.

Para efectuar este modelo es importante involucrar a las diferentes unidades académicas que pueden sustentar su elaboración, como son las escuelas, la unidad especializada de evaluación y la investigación. Lo anterior promovería la realización de estudios periódicos, pero con carácter permanente como serían: análisis de las cargas académicas; evaluación de aprendizajes y tutorías presenciales; prueba de nuevas tecnologías de acuerdo a sus potencialidades pedagógicas y acordes con el modelo pedagógico. Ello permitiría la orientación de la inversión tecnológica y el desarrollo de planes pilotos. Dichos planes pilotos se harían con el fin de cambiar el esquema en la utilización de la tecnología en el área académica, de tal forma que se centre la atención en la búsqueda de tecnologías que se ajusten a las necesidades de aprendizaje de los cursos y no viceversa.

Adicionalmente se recomienda realizar un estudio permanente de seguimiento a graduados, que permita determinar dónde se ubican y algunos parámetros sobre el grado de éxito o fracaso que éstos han tenido.

El modelo lo deben implementar totalmente las escuelas, con asesorías pedagógicas de otras dependencias, pero los expertos en contenido deben ser los docentes en sus respectivas especialidades, los cuales, teniendo una equitativa carga administrativa-académica, podrán realizar estas otras actividades académico didácticas de más trascendencia.

A continuación se presenta el diagrama \#3 que resume los aspectos fundamentales tratados en la investigación aquí referida. 
DIAGRAMA NO. 3

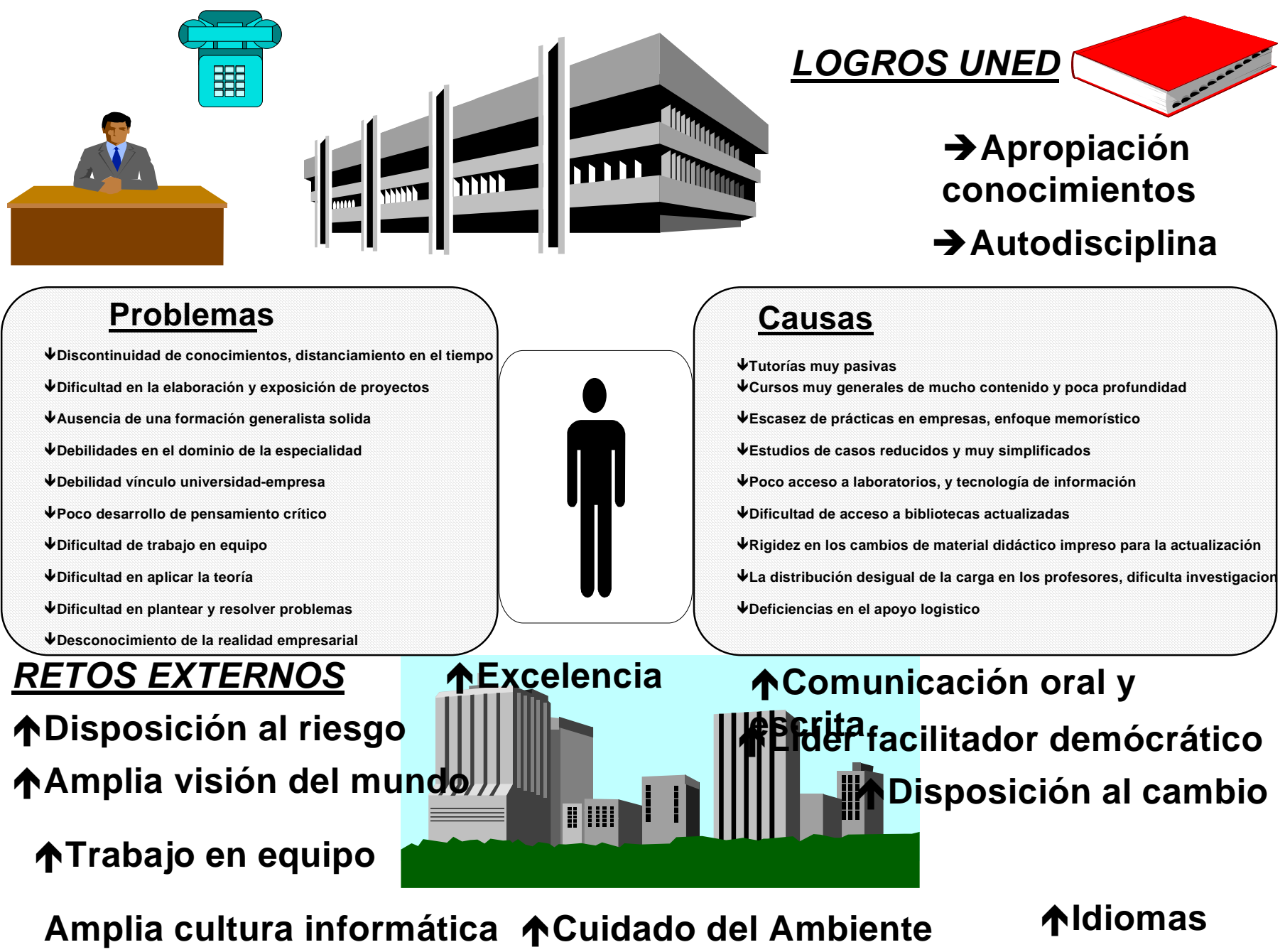




\section{REFERENCIAS BIBLIOGRÁFICAS}

Ahumada A., Pedro. S.F. (). Indicadores de calidad académica: punto de partida en una evaluación curricular. Chile: Universidad Católica del Valparaíso. Material mimeografiado.

CARTAS, José M. (1997). Los desafíos planteados por la globalización a los mercados laborales en América Latina, en Seminario internacional "Los mercados laborales eficientes: Un desafío para América Latina. San José, Costa Rica: Fundación Konrad Adenauer- CIEDLA (Centro Interdisciplinario de Estudios sobre el Desarrollo Latinoamericano) y ICEP (Instituto Costarricense de Estudios Políticos. 29 y 30 de julio

CONSEJO NACIONAL DE RECTORES - OFICINA DE PLANIFICACIÓN DE LA EDUCACIÓN SUPERIOR (CONAREOPES) (1995). Posibilidades de estudio en la educación superior universitaria estatal de Costa Rica. Costa Rica: Opes 05/95.

(1996). Estadísticas de la Educación Superior 1993 y 1994. Costa Rica: Opes 06/96.

$\left(1996^{a}\right)$. Lineamientos para la acreditación de carreras y programas de la educación superior. Costa Rica: Comisión interinstitucional. Noviembre. Documento mimeografiado.

(1996 $6^{\text {b }}$. Lineamientos para la acreditación de carreras y programas de la educación superior. Aspectos, criterios e indicadores de la calidad académica. Costa Rica: Comisión interinstitucional. Documento mimeografiado.

(1996 $)$. Lineamientos para la acreditación de carreras y programas de la educación superior. Resumen Ejecutivo. Costa Rica: Comisión interinstitucional. Documento mimeografiado.

CONSEJO SUPERIOR UNIVERSITARIO CENTROAMERICANO (CSUCA). S.F. (). Nueva Propuesta. "Sistema centroamericano de evaluación y acreditación de la educación superior (SICEVAES). (Primer borrador para discusión). Costa Rica. Documento mimeografiado

Chase, B.R., GARvin, A.D. (1989). The Service Factory. Harvard-Deusto, Business Review. Julio-agosto.

Cruz, A., Hidalgo, G. y CAstillo, A. (1997). Propuesta para mejorar el programa de Administración de Empresas. Costa Rica: UNED.

DruCKER, P. (1988). Coming of the new Organization. Harvard-Deusto, Business Review. Enero-febrero.

Escotet, M. A. (1992). Aprender para el futuro. Madrid: Editorial Alianza Universidad.

ESPINOZA, O. Y OTROS (1994). Manual autoevaluación para instituciones de educación superior. Pautas y procedimientos. CINDA/PROMESUP/OEA. Chile.

GüELL, A. (1996). El directivo del futuro. Harvard-Deusto Business Review.

L'ECUYER, J. (1997). La evaluación de programas. Material entregado en la Conferencia “Autoevaluación y acreditación, San José, Costa Rica: Instituto Tecnológico de Costa Rica, 2 de julio.

MCKenNA R. (1991). Marketing is everything. Harvard Business Review. Enero-febrero.

PuryeAR, J. (1996). Education in Latin America: problems and Challenges. Working Group on Educational Reform. Council on Foreign Relations. New York, N.Y. http://foreignaffairs.org/conference/puryear.html

RAMíREZ, H. (1997). Materiales, evaluación y tutorías en los cursos de la UNED de Costa Rica, en: Revista Innovaciones Educativas, 4 (7). Costa Rica.

Rojo, J. M. (1997). Universidad y Sociedad: los retos de la reforma universitaria. Costa Rica: Conferencia en la Sala de Ex Rectores, Biblioteca Joaquín García Monge, Universidad Nacional, 8 agosto.

RuIZ, O., José L. E IsPIZUA, M.A. (1989). La descodificación de la vida cotidiana. Métodos de investigación cualitativa. Bilbao: Universidad de Deusto.

SINGH, A. (1997). Globalización y desarrollo sostenible: el reto del empleo. Conferencia: El desarrollo humano sostenible frente a la globalización. San José, Costa Rica: Ministerio de Planificación y política económica-PNUD, 28 y 29 de agosto.

StalK, G. (1988). Time-The next source of competitive advantage. Harvard Business Review. Julio-agosto.

TOFFleR, A. (1994). La creación de una nueva civilización. Editorial Norma, Bogotá. Colombia.

UNIVERSIDAD Estatal a DistAnCIA (1996). Informe de labores. La UNED hacia el siglo XXI 1986-1996. Presentado a la Asamblea Universitaria por el Dr. Celedonio Ramírez R. Rector. Costa Rica: UNED, mayo.

$\left(1996^{a}\right)$. Análisis y evaluación del plan anual operativo y su relación con el presupuesto al 30 de junio de 1996. Costa Rica: Oficina de programación y análisis administrativo, UNED, noviembre. 


\section{PERFIL ACADÉMICO-PROFESIONAL DE LAS AUTORAS}

Alejandra Cruz Muñoz es licenciada en Sociología. Investigadora del Centro de Investigación Académica (CIAC) de la UNEDde Costa Rica. Experiencia en producción y evaluación de materiales didácticos, evaluación de programas, investigación sobre estudiantes y graduados.

Teléfono: (506) 253-2121, ext. 2242

Fax: (506) 234-1909

E Mail: acruz@arenal.uned.ac.cr

Guiselle Hidalgo Molina es licenciada en Estadística, Master en Econometría. Investigadora del Centro de Investigación y Evaluación Institucional (CIEI) de la UNEDde Costa Rica. Experiencia en aplicación de la tecnología de información en educación a distancia y evaluación de programas.

Teléfono: (506) 253-2121, ext. 2241

Fax: (506) 234-1909

E Mail: ghidalgo@arenal.uned.ac.cr.

Anabelle Castillo López es licenciada en Administración Pública, Master en Administración de Empresas. Investigadora-Docente en la escuela de Administración de la UNED de Costa Rica. Experiencia en adaptar recursos tecnológicos a cursos específicos y evaluación de programas.

Teléfono: (506) 234-1909

Fax: (506) 256-4473

E Mail: acastill@arenal.uned.ac.cr 